Luís Antônio Benvegnú

Anaclaudia Gastal Fassa²

Luiz Augusto Facchini ${ }^{3}$

Fabiana Breitenbach ${ }^{4}$

\section{Prevalência de hipertensão arterial entre motoristas de ônibus em Santa Maria, Rio Grande do Sul}

\author{
Hypertension prevalence among bus drivers in Santa Maria, Rio
}

Grande do Sul, Brazil
${ }^{1}$ MD, MSc, PhD, Departamento de Ciências da Saúde da Universidade Regional do Noroeste do Estado do Rio Grande do Sul (Unijuí).

${ }^{2} \mathrm{MD}, \mathrm{MSc}, \mathrm{PhD}$, Departamento de Medicina Social da Universidade Federal de Pelotas (UFPel). Rio Grande do Sul.

${ }^{3} \mathrm{MD}, \mathrm{MSc}, \mathrm{PhD}$, Departamento de Medicina Social - Universidade Federal de Pelotas (UFPel). Rio Grande do Sul.

${ }^{4} \mathrm{MD}$, Fundação Municipal de Saúde de Santa Rosa. Rio Grande do Sul.

Contato:

Luís Antônio Benvegnú

Rua Doralino Leusin, 126 - Santa Rosa, RS

CEP 98.900-000

E-mail:

luis.benvegnu@unijui.edu.br

O projeto recebeu apoio da Fundação de Apoio a Pesquisa do Rio Grande do Sul (FAPERGS).

Recebido: 11/09/2007

Revisado: $31 / 07 / 2008$

Aprovado: $11 / 08 / 2008$

\section{Resumo}

Objetivos: O objetivo deste estudo foi identificar a prevalência de hipertensão arterial sistêmica (HAS) em motoristas de ônibus urbanos de Santa Maria/RS e estudar fatores associados à mesma nesta categoria profissional. Metodologia: Realizou-se um estudo epidemiológico do tipo transversal com 214 motoristas e igual número de vizinhos, selecionados por sexo e idade. Foi utilizado um questionário individual padronizado e realizadas medidas da pressão arterial, peso e altura no domicílio do entrevistado. Resultados: A prevalência de HAS entre os motoristas foi de $22,4 \%$, não apresentando diferença significativa entre os grupos, mesmo controlados os fatores de confusão. Entretanto, entre os motoristas, a obesidade (RP 2,30 IC95\% 1,04-5,07) e os problemas psiquiátricos menores (RP 2,26 IC95\% 1,15-4,43) estiveram associados à HAS. Conclusões: O fato de Santa Maria ser uma cidade do interior, com um trânsito menos intenso e de menor complexidade pode ser uma explicação para que os motoristas não apresentem maior prevalência de HAS do que seus vizinhos. O perfil de classe parece ser mais importante do que as características específicas do trabalho de dirigir ônibus.

Palavras-chave: hipertensão arterial sistêmica, motoristas de ônibus, saúde do trabalhador, epidemiologia, obesidade.

\begin{abstract}
Objectives: The goal of this study was to verify the prevalence and to study associations between hypertension and bus drivers' professional activity. Methods: A cross sectional study was conduced in the town of Santa Maria, involving 214 urban bus drivers and the same number of their neighbors, selected by sex and age. A standardized questionnaire was applied to each individual and their arterial tension, weight and height were measured at their home. Results: The hypertension prevalence among bus drivers was $22.4 \%$. A significant difference was not observed with the control group, even after confounders were controlled. However, among drivers, obesity (RP 2.30 IC95\% 1.04-5.07) and minor psychiatric disorders (RP 2.26 IC95\%1.15-4.43) were associated to hypertension. Conclusions: The fact of Santa Maria being located in the country side, without such intense and complex traffic, can be the explanation for the drivers not presenting a greater prevalence of hypertension when compared to their neighbors. The class profile probably surpasses bus drivers' professional activity in hypertension determination.
\end{abstract}

Keywords: hypertension, bus drivers, occupational health, epidemiology, obesity. 


\section{Introdução}

A categoria dos motoristas de ônibus urbanos tem grande importância social, principalmente nas sociedades contemporâneas e mais urbanizadas, não só pela exposição a condições de trabalho bastante específicas, mas também pela responsabilidade coletiva de sua atividade: o transporte cotidiano de passageiros. Em função disso, este grupo vem sendo objeto freqüente de estudos epidemiológicos na área de saúde do trabalhador e da medicina ocupacional.

A hipertensão arterial sistêmica (HAS) destaca-se como um dos principais problemas de saúde pública no Brasil, especialmente nas regiões Sul e Sudeste do país, incidindo em $22,3 \%$ a $43,9 \%$ da população adulta (DÓREA; LOTUFO, 2004; GUS et al., 2004). Além de altamente prevalente, é importante fator de risco para doença coronariana, uma das principais causas de morte em adultos em idade produtiva (PICCINI, 1993).

Winkleby et al. (1988a), numa revisão de 22 artigos, identificaram excesso de risco para morbidade e mortalidade entre os motoristas de ônibus. Os problemas gástricos, musculoesqueléticos e cardíacos foram os mais fortemente associados com a tarefa de dirigir ônibus. Foram relatados, em estudos realizados no Brasil, distúrbios no sono, sedentarismo, ansiedade (MELLO et al., 2000; SANTOS et al., 2004; OLIVEIRA; PINHEIRO, 2007), problemas psiquiátricos menores (SOUZA; SILVA, 1998), hipertensão, obesidade, problemas osteomusculares e gastrointestinais (COSTA et al., 2003), entre outros.

A ocorrência de doenças cardíacas isquêmicas e hipertensão em motoristas de ônibus tem sido tema de vários estudos. São relatadas evidências de excesso de risco destas doenças para os motoristas quando comparados com trabalhadores de outros setores da mesma empresa ou com outras categorias ocupacionais (NETTERSTROM; SUADICANI, 1993; HARTVIG; MIDTTUN, 1983; WINKLEBY et al., 1988b; ALFREDSSON; HAMMAR; HOGSTEDT, 1993; MICHAELS; ZOLOTH, 1991; ROSENGREN; ANDERSNO; WILHELMSEN, 1991; WANG; LIN, 2001; MORRIS et al., 1966; CORDEIRO et al., 1993; CORREA FILHO et al., 2002).

Os achados para hipertensão, entretanto, apresentam alguns resultados controversos. Vários estudos não controlam fatores de confusão e o grupo de comparação, muitas vezes, é formado por trabalhadores de uma única categoria, o que limita a generalização dos achados (AHUMADA; RAMIREZ, 1991; BACKMAN; JARVINEN, 1983; HARTVIG; MIDTTUN, 1983; RAGLAND et al., 1997; WANG; LIN, 2001). Ragland et al. (1997), estudando HAS em motoristas de ônibus urbanos em São Francisco, observaram prevalência de 38,9\% entre sujeitos com mais de 20 anos de trabalho e $28,8 \%$ em trabalhadores ingressando na profissão, diferença significante mesmo após controlar por fatores como raça, idade e índice de massa corporal. Rosengren et al. (1991), contudo, apesar de encontrarem maior incidência de doença coronariana em motoristas, comparados com trabalhadores de várias outras profissões, não encontraram diferenças significativas nos níveis pressóricos verificados em ambos os grupos.
O objetivo deste estudo foi verificar a prevalência de hipertensão entre os motoristas de ônibus urbanos da cidade de Santa Maria-RS e os fatores associados à HAS nestes trabalhadores quando comparados com uma amostra dos seus vizinhos, controlando os fatores de confusão e identificando fatores de risco que facilitem o controle deste problema de saúde neste grupo ocupacional.

\section{Metodologia}

Utilizou-se delineamento transversal, selecionando-se, para cada motorista de ônibus urbano, um vizinho que estivesse trabalhando com outra profissão e que tivesse a mesma idade ( \pm 5 anos), com definição a priori de expostos (motoristas) e não expostos (vizinhos) (KLEINBAUM et al., 1998). O estudo foi realizado na cidade de Santa Maria, localizada na região central do Rio Grande do Sul, com uma população de aproximadamente 240 mil habitantes (INSTITUTO BRASILEIRO DE GEOGRAFIA E ESTATÍSTA, 2001).

No cálculo do tamanho da amostra, utilizaram-se os seguintes parâmetros estatísticos: nível de confiança de $95 \%$, poder estatístico de $80 \%$, prevalência de hipertensão nos vizinhos de $18 \%$ e razão de prevalências de 1,8, obtendo-se uma amostra de 218 motoristas e 218 vizinhos após acrescidos $10 \%$ para possíveis perdas e $30 \%$ para controle de fatores de confusão.

Obteve-se uma lista com nome e endereço dos 222 motoristas de ônibus urbanos nas cinco empresas da cidade. Destes, três recusaram-se a responder ao questionário e cinco não foram localizados, resultando numa perda de 3,6\%.

Aplicou-se um questionário padronizado e pré-codificado para cada motorista e para o primeiro vizinho que atendesse aos critérios citados, começando-se pelo domicílio à direita da residência do motorista. Uma vez identificado um vizinho, retornou-se tantas vezes quanto necessário para realizar a entrevista, não sendo substituído em nenhum caso. Foram obtidas informações demográfico-comportamentais, como idade, cor da pele, escolaridade, estado civil, tabagismo, alcoolismo - verificado através do teste CAGE (BEIGEL et al., 1974) -, problemas psiquiátricos - medidos através do teste SRQ20 (MARI; WILLIAMS, 1986) -, atividade física e Índice de Massa Corporal - peso/altura ${ }^{2}$, peso em quilogramas e altura em metros (ANJOS, 1992). Também foram coletadas informações em relação às características ocupacionais, como: função, renda mensal, antiguidade na função, duração da jornada e turno de trabalho.

O peso e a estatura foram medidos através de balanças de banheiro, calibradas diariamente, e de antropômetros tipo régua confeccionados especialmente para a pesquisa, sendo o entrevistado medido em pé e descalço. A pressão arterial foi medida pelos próprios entrevistadores no final da entrevista. A medida foi realizada no braço direito com o indivíduo sentado e o braço à altura da região mamária, utilizando-se esfigmomanômetro aneróide calibrado semanalmente. Foram considerados hipertensos aqueles que apresentaram pressão arterial sistólica acima de $160 \mathrm{mmHg}$ e/ou pressão diastólica igual ou superior a $95 \mathrm{mmHg}$ e mais 
os que relataram hipertensão e que faziam uso de medicamentos independentemente da medida obtida.

As entrevistas foram realizadas no domicílio, visando facilitar a localização do vizinho e padronizar o local da coleta de dados. Os entrevistadores foram estudantes de medicina criteriosamente treinados para esta pesquisa. Para controle de qualidade, eram realizadas entrevistas acompanhadas e foram feitas revisitas em $5 \%$ da amostra, não evidenciando problemas com a coleta de dados.

A análise inicial incluiu o cálculo das medidas de freqüência e distribuição das variáveis para os motoristas e vizinhos. A medida de efeito utilizada foi razão de prevalências (RP), com o respectivo intervalo de confiança de $95 \%$, e o teste estatístico do qui-quadrado.

A análise multivariada foi realizada por regressão de Poisson, através do programa Stata. A significância estatística foi obtida através do teste da razão de verossimilhança. Para controle de fatores de confusão, foram levadas para a análise as variáveis associadas à ocupação e à hipertensão com p<0,2.

Os entrevistados prestaram consentimento verbal informado para responder o questionário e realizar as medidas citadas. Foi garantida a confidencialidade das informações prestadas e o direito a não responder. As pessoas que apresentavam alguma alteração eram encaminhadas para assistência médica.

\section{Resultados}

Foram entrevistados 214 motoristas e 214 vizinhos. A média de idade dos motoristas e vizinhos foi semelhante, 37,6 anos ( $\mathrm{dp}=8,0$ ), e a grande maioria era de cor branca em ambos os grupos. Contudo, os motoristas apresentavam menor grau de escolaridade: $50,5 \%$ havia estudado até cinco anos, enquanto quase a metade dos vizinhos havia completado nove anos ou mais de estudo.

A prevalência de hipertensão foi semelhante entre motoristas e vizinhos, $22,4 \%$ e $24,3 \%$, respectivamente. Também não foram observadas diferenças quanto ao hábito de fumar e a prevalência de problemas psiquiátricos. Todavia, o alcoolismo foi significativamente mais freqüente entre os vizinhos e o sedentarismo e a obesidade, entre os motoristas $(\mathrm{p}<0,05)$ (Tabela 1).

Tabela 1 Distribuição das principais variáveis entre motoristas e vizinhos Santa Maria, RS

\begin{tabular}{|c|c|c|c|c|}
\hline \multirow{2}{*}{ Variável } & \multicolumn{2}{|c|}{ Motoristas } & \multicolumn{2}{|c|}{ Vizinhos } \\
\hline & $n$ & $\%$ & $n$ & $\%$ \\
\hline \multicolumn{5}{|l|}{ Hipertensão arterial sistêmica } \\
\hline Não & 166 & 77,6 & 162 & 75,7 \\
\hline Sim & 48 & 22,4 & 52 & 24,3 \\
\hline \multicolumn{5}{|c|}{ Realizou exercício físico no último ano* } \\
\hline Não & 138 & 64,5 & 115 & 53,7 \\
\hline Até 2 x semana & 56 & 26,2 & 63 & 29,4 \\
\hline $3 \times$ semana ou + & 20 & 9,3 & 36 & 16,8 \\
\hline \multicolumn{5}{|l|}{ Índice de massa corporal* } \\
\hline Normal $<=24$ & 80 & 37,4 & 124 & 57,9 \\
\hline Sobrepeso 25-29 & 87 & 40,7 & 60 & 28,0 \\
\hline Obeso 30 ou + & 47 & 22,0 & 30 & 14,0 \\
\hline \multicolumn{5}{|l|}{ Problemas psiquiátricos menores } \\
\hline SRQ-20 positivo & 26 & 12,1 & 37 & 17,3 \\
\hline SRQ-20 negativo & 188 & 87,9 & 177 & 82,7 \\
\hline \multicolumn{5}{|l|}{ Insônia* } \\
\hline Não & 167 & 78,0 & 134 & 66,6 \\
\hline Sim & 47 & 22,0 & 80 & 37,4 \\
\hline \multicolumn{5}{|l|}{ Alcoolismo* } \\
\hline CAGE positivo & 9 & 4,2 & 22 & 10,3 \\
\hline CAGE negativo & 205 & 95,8 & 192 & 89,7 \\
\hline \multicolumn{5}{|l|}{ Antiguidade na função* } \\
\hline Até 10 anos & 87 & 40,6 & 110 & 51,4 \\
\hline 10 anos ou + & 127 & 59,4 & 104 & 48,6 \\
\hline \multicolumn{5}{|l|}{ Turno de trabalho* } \\
\hline Diurno & 150 & 70,1 & 172 & 80,4 \\
\hline Misto & 64 & 29,9 & 42 & 19,6 \\
\hline
\end{tabular}

" Diferença estatisticamente significativa entre motoristas e vizinhos, $p<0,05$

SM = salário mínimo

n = motoristas: 214; vizinhos: 214 . 
Dentre as ocupações dos vizinhos, houve predomínio de trabalhadores no comércio e em prestação de serviços ao público (46,7\%), seguidos de trabalhadores diretos no processo de produção industrial, inclusive construção civil (20,6\%), artesãos, fabricantes e comerciantes em pequena escala $(15,4 \%)$, profissionais de nível superior e técnicos especializados (10,7\%). Aproximadamente três em cada quatro motoristas percebia renda mensal entre 2,6 e 4,0 salários mínimos, enquanto os vizinhos constituíram dois grupos principais, um acima e o outro abaixo desta faixa $(p<0,05)$.

Observou-se que cerca de $60 \%$ dos motoristas e $49 \%$ dos vizinhos apresentavam mais de dez anos de antiguidade na função $(\mathrm{p}<0,05)$. A proporção de trabalhadores em turno misto (parte diurno e parte noturno ou rotativo) foi significativamente maior que a dos vizinhos (Tabela 1).

As variáveis demográficas e comportamentais bem como as ocupacionais foram testadas para associação com HAS. Para os motoristas, as variáveis que se mos- traram associadas foram o índice de massa corporal, que apresentou RP 1,92 (IC95\% 0,94-3,94) para o sobrepeso e 2,17 (IC95\% 0,99-4,78) para os obesos, e problemas psiquiátricos menores, com RP 2,15 (IC95\% 1,26-3,67). O alcoolismo com RP de 1,67 (0,92-3,03) e o sedentarismo, quando a com RP de quem não tinha o hábito de fazer exercícios foi 2,61 (IC95\% 0,63-10,8) em relação a quem fazia pelo menos três vezes por semana, apresentaram valor $\mathrm{p}<0,2$ e por isso foram testadas na análise multivariada. Entre os vizinhos, os obesos também apresentaram maior prevalência de hipertensão do que os com IMC normal, RP 3,70 (IC95\% 1,92-7,11), e os que estavam no emprego há mais de 10 anos apresentaram RP 1,56 (IC95\% 0,962,54) (Tabela 2).

Entre os motoristas, as variáveis que se mantiveram associadas com hipertensão na análise multivariada foram IMC e problemas psiquiátricos menores, que apresentaram pequenas variações nas razões de prevalência em relação aos valores brutos, evidenciando o efeito independente dessas características na prevalência de HAS (Tabela 3).

Tabela 2 Fatores de risco para hipertensão arterial sistêmica: análise bruta entre motoristas de ônibus e seus vizinhos, Santa Maria, RS

\begin{tabular}{|c|c|c|c|c|c|c|}
\hline \multirow[t]{2}{*}{ Variável } & \multicolumn{3}{|c|}{$\begin{array}{c}\text { Motoristas } \\
n=214\end{array}$} & \multicolumn{3}{|c|}{$\begin{array}{l}\text { Vizinhos } \\
n=214\end{array}$} \\
\hline & prev $\%$ & $R P$ & IC 95\% & prev\% & $R P$ & IC 95\% \\
\hline \multicolumn{7}{|l|}{ Índice de massa corporal } \\
\hline Normal $<=24$ & 13,8 & 1 & $*$ & 15,3 & 1 & * \\
\hline Sobrepeso 25-30 & 26,4 & 1,92 & $0,94-3,94$ & 26,7 & 1,74 & $0,89-3,38$ \\
\hline Obeso $>30$ & 29,8 & 2,17 & $0,99-4,78$ & 56,7 & 3,70 & $1,92-7,11$ \\
\hline \multicolumn{7}{|c|}{ Problemas psiquiátricos menores } \\
\hline SRQ-20 negativo & 19,7 & 1 & & 25,4 & 1 & \\
\hline SRQ-20 positivo & 42,3 & 2,15 & $1,26-3,67$ & 18,9 & 0,74 & $0,36-1,51$ \\
\hline \multicolumn{7}{|l|}{ Alcoolismo } \\
\hline CAGE negativo & 20,7 & 1 & & 23,3 & 1 & \\
\hline CAGE positivo & 34,6 & 1,67 & $0,92-3,03$ & 28,6 & 1,23 & $0,71-2,13$ \\
\hline \multicolumn{7}{|l|}{ Exercício físico } \\
\hline $3 \times$ semana ou mais & 10,0 & 1 & & 22,2 & 1 & \\
\hline Até 2 x semana & 17,9 & 1,79 & $0,39-8,15$ & 19,1 & 0,86 & $0,35-2,01$ \\
\hline Não faz & 26,1 & 2,61 & $0,63-10,8$ & 27,8 & 1,25 & $0,58-2,71$ \\
\hline \multicolumn{7}{|l|}{ Ocupacionais } \\
\hline \multicolumn{7}{|c|}{ Antiguidade na função } \\
\hline Até 10 anos & 20,1 & 1 & & 19,1 & 1 & \\
\hline 10 anos ou + & 23,6 & 1,14 & $0,68-1,91$ & 29,8 & 1,56 & $0,96-2,54$ \\
\hline \multicolumn{7}{|l|}{ Turno de trabalho } \\
\hline Diurno & 22,7 & 1 & & 23,3 & 1 & \\
\hline Misto & 21,8 & 0,96 & $0,56-1,67$ & 28,6 & 1,22 & $0,71-2,13$ \\
\hline
\end{tabular}

* p da tendência linear $<0,05$

$\mathrm{RP}=$ razão de prevalências

IC = intervalo de confiança 
Tabela 3 Fatores de risco para hipertensão arterial sistêmica entre motoristas de ônibus, Santa Maria, RS, $n=214$

\begin{tabular}{|c|c|c|c|c|}
\hline \multirow[b]{3}{*}{ Variável } & \multirow{3}{*}{$\begin{array}{c}\text { prev HAS } \\
\% \\
\end{array}$} & \multicolumn{3}{|c|}{ Regressão de Poisson } \\
\hline & & \multicolumn{3}{|c|}{ Ajustado } \\
\hline & & $R P$ & IC $95 \%$ & $p$ \\
\hline \multicolumn{5}{|l|}{ Índice de massa corporal } \\
\hline Normal $<=24$ & 13,8 & 1 & & \\
\hline Sobrepeso 25-30 & 26,4 & 1,95 & $0,95-4,00$ & 0,069 \\
\hline \multirow[t]{2}{*}{ Obeso $>30$} & 29,8 & 2,30 & $1,04-5,07$ & 0,040 \\
\hline & & & Tendência linear & 0,034 \\
\hline \multicolumn{5}{|l|}{ Problemas psiquiátricos menores } \\
\hline SRQ-20 negativo & 19,7 & 1 & & \\
\hline SRQ-20 positivo & 42,3 & 2,26 & $1,15-4,43$ & 0,018 \\
\hline
\end{tabular}

\section{Discussão}

No presente estudo não se encontrou maior prevalência de HAS nos motoristas de ônibus urbanos da cidade de Santa Maria quando comparados com uma amostra de seus vizinhos, mesmo após o controle de fatores de confusão.

Entretanto, à semelhança de outros estudos realizados na região (PICCINI, 1993; GUS et al., 2004), a prevalência observada nos motoristas foi elevada, $22,4 \%$, confirmando a HAS como um dos mais importantes problemas de saúde desta categoria ocupacional e de saúde pública. Esta constatação é reforçada por estudos que envolveram motoristas de ônibus de diferentes lugares, os quais mostram prevalências entre 20\% e 47\% (AHUMADA; RAMIREZ, 1991; BACKMAN; JARVINEN, 1983; CORDEIRO et al., 1993; COTTINGTON et al., 1985; RAGLAND et al., 1997; WANG; LIN, 2001; COSTA et al., 2003).

Estes estudos evidenciaram excesso de risco para hipertensão arterial entre os motoristas utilizando diferentes grupos para comparação. Backman (1983) realizou comparações com outros motoristas profissionais na Finlândia. Ahumada e Ramirez (1991) selecionaram trabalhadores da manutenção e da administração de uma mesma empresa de transporte na Cidade do México para comparação. Ragland et al. (1997) utilizaram três grupos: trabalhadores de diferentes ocupações, trabalhadores das mesmas áreas geográficas e pretendentes ao emprego de motoristas de ônibus, em São Francisco, EUA. Diversos estudos verificaram a pressão arterial como fator de risco para as doenças cardíacas e também encontraram risco aumentado entre os motoristas (HARTVIG; MIDTTUN, 1983; MARTINS et al., 2003; MORRIS et al., 1966; WANG; LIN, 2001; NETTERSTROM; SUADICANI, 1993; BIGERT et al., 2003).
Por outro lado, Rosengren, Anderson e Wilhelmsen (1991), através de um estudo prospectivo, com seguimento de cerca de doze anos, delineado para investigar fatores de risco para doença cardiovascular, não encontraram risco aumentado de HAS em motoristas quando comparado e com outras profissões, apesar de ter evidenciado, no mesmo trabalho, risco 3,3 vezes maior de doença coronariana para os motoristas. Cabe salientar que a população no final do acompanhamento era composta por homens com idade entre 47 e 55 anos. Bigert et al. (2003), baseados nos resultados do estudo em Estocolmo, sugerem que o risco aumentado entre os motoristas de ônibus pode ser parcialmente explicado pelo estilo de vida dos trabalhadores, além das exposições ocupacionais.

Antes de entrar na discussão deste tema, em que a própria literatura apresenta resultados controversos, convém examinar alguns aspectos metodológicos deste estudo. A realização da coleta de dados no domicílio, tanto para o grupo de motoristas, quanto para o de vizinhos, utilizando instrumentos padronizados e equipe criteriosamente treinada, são aspectos que reforçam a acurácia das medidas efetuadas. A seleção de indivíduos do mesmo estrato social que os motoristas, mas com outras ocupações, como grupo de comparação parece bastante adequada para se avaliar a participação do trabalho na determinação da hipertensão arterial. Além disso, ao se utilizar um controle populacional, ao invés de uma categoria ocupacional específica, amplia-se a capacidade de generalização dos achados.

Entretanto, por tratar-se de um estudo transversal, a possibilidade de "efeito do trabalhador sadio" (CHECKOWAY; PERACE; KRIEBEL, 2004) deve ser considerada, pois a população de motoristas poderia ser composta por indivíduos que superaram ou se adaptaram às dificuldades apresentadas pelo trabalho de dirigir ônibus urbanos. Desta forma, a saída dos menos saudáveis contribuiria para minimizar as diferenças entre motoristas e vizinhos. Entre- 
tanto, este efeito parece não ter ocorrido neste estudo, considerando que os motoristas mais antigos apresentaram maiores prevalências de hipertensão, sugerindo a inexistência de um afastamento importante de trabalhadores por este problema de saúde. Além disso, em cidades menores, o trabalho como motorista de ônibus oportuniza melhores salários e maior reconhecimento social para a escolaridade exigida, o que também reforça o vínculo ocupacional destes trabalhadores.

O fato de Santa Maria ser uma cidade do interior, menor do que a maioria das cidades onde foram realizados os demais estudos, com um trânsito menos intenso e de menor complexidade pode ser uma explicação para que os motoristas não apresentem maior prevalência de HAS quando comparados com seus vizinhos. Neste sentido, Netterstrom e Suadicani (1993) observaram que os condutores dinamarqueses que cumpriam itinerários no centro da cidade apresentavam maior risco de infarto agudo do miocárdio em relação aos que trabalhavam com itinerários suburbanos. Os autores sugerem que o maior contato com os passageiros e a intensidade do tráfego são as principais diferenças entre os itinerários. Alfredson et al. (1993) constataram um incremento na mortalidade por infarto do miocárdio e doenças cardíacas isquêmicas e também na taxa de incidência de infarto do miocárdio em motoristas suecos nas regiões que englobam as grandes cidades do país. Não observou o mesmo nas regiões com predomínio de áreas rurais. Os grandes centros urbanos oferecem um ambiente favorável ao estresse e a outros fatores psicossociais que interferem na prevalência de HAS e doenças coronarianas (ISSEVER et al., 2002; RYDSTEDT; JOHANSSON; EVANS, 1998; SOUZA; SILVA, 1998; COTTINGTON et al., 1985) com reflexos aparentemente mais graves sobre os motoristas de ônibus. $\mathrm{O}$ relacionamento mais próximo e personalizado com os patrões, por se tratarem de empresas de pequeno porte, também pode evitar momentos de maior estresse (COSTA et al., 2003). Além disso, nas cidades menores, os motoristas parecem gozar de um melhor status socioeconômico em relação a seu estrato social, cujas possíveis influências positivas sobre a saúde também reforçariam os achados do presente estudo.

Contudo, entre os motoristas de Santa Maria, observamos uma associação significativa entre os problemas psiquiátricos menores e a hipertensão. Este achado parece reforçar a hipótese da associação entre estresse e problemas psicológicos com hipertensão. Assim, em função das características da cidade e do tráfego, os motoristas não têm mais problemas psicológicos do que os vizinhos, mas, entre os motoristas com problemas psicológicos, a hipertensão é mais prevalente do que nos demais.

Neste caso, a reação do indivíduo ao estresse no cotidiano do trabalho resultaria em sofrimento psíquico que poderia contribuir para a ocorrência de HAS.
Diversos estudos têm sido realizados evidenciando a presença e a importância dos problemas psicológicos em motoristas de ônibus, a presença de fatores estressantes no seu trabalho (MARTINS et al., 2003; PAESMACHADO; LEVENSTEIN, 2002; SOUZA; SILVA, 1998; WAHLBERG, 2004; BENAVIDES et al., 2003; VANDERVOORT; RAGLAND; SYME, 2001; MELLO et al., 2000; SANTOS et al., 2004; OLIVEIRA; PINHEIRO, 2007) e a validade da coleta dessas informações através de questionários em estudos epidemiológicos (GIMENO et al., 2004). A Razão de Prevalências de HAS em relação ao alcoolismo foi de 1,67 (IC95\% 0,92-3,03). A literatura refere problemas de atenção decorrentes de alterações no sistema nervoso central provocados pelo consumo excessivo de bebidas alcoólicas, o que pode estar contribuindo para o aumento da prevalência de problemas psicológicos e, conseqüentemente, para o aumentando da prevalência de HAS e do risco de acidentes (GARRIDO; FERNANDEZ-GUINEA, 2004; MELLO et al., 2000; COSTA et al., 2003).

A idade e a obesidade estiveram associadas significativamente com a hipertensão, a exemplo do encontrado em São Paulo e Belo Horizonte (COSTA et al., 2003). Os demais fatores de risco classicamente associados com HAS, como escolaridade, renda, sedentarismo, ruído e calor (CORDEIRO; LIMA FILHO; NASCIMENTO, 1994; CORREA FILHO et al., 2002; KLOETZEL et al., 1973; LÓLIO et al., 1993; PICCINI, 1993), possivelmente em função da relativa homogeneidade da população estudada, não mostraram associação com hipertensão neste estudo. A idade foi utilizada como critério de seleção do grupo de controle e, portanto, não foi inserida na análise multivariada quando incluía os dois grupos.

A obesidade destacou-se como a variável mais fortemente associada com HAS, elevando a prevalência entre os obesos para mais do que o dobro daqueles com peso dentro dos níveis de normalidade. Assim, a obesidade, por ser evitável, destaca-se como um dos principais fatores a serem abordados na prevenção da hipertensão arterial sistêmica entre motoristas, a exemplo do relatado por Wang e Lin (2001).

Portanto, programas de controle de hipertensão entre os motoristas de ônibus, necessários em função da alta prevalência do problema, deverão levar em conta estratégias de diminuição da obesidade, prevenção dos problemas psiquiátricos e diminuição do estresse nesta profissão. Como o sedentarismo foi evidenciado em cerca de dois terços dos membros desta categoria ocupacional, o aumento da atividade física, por exemplo, com atividades orientadas nos intervalos, no próprio ambiente de trabalho, parecem ser uma boa alternativa. No entanto, estudos com delineamento longitudinal são necessários para detalhar os mecanismos etiológicos da HAS entre os motoristas de ônibus. 


\section{Agradecimentos}

Este trabalho contou com apoio da Fundação de Apoio à Pesquisa do Estado do Rio Grande do Sul, através de bolsa de iniciação científica. Agradecemos também aos motoristas e vizinhos que participaram do estudo e aos entrevistadores.

\section{Referências}

AHUMADA, H.; RAMIREZ, F. L. Las condiciones de salud de los conductores de autotransportes urbanos de pasajeros de la ciudad de México. B. Oficina Sanit. Panam., Washington, v. 111, n. 6, p. 324-332, dic. 1991.

ALFREDSSON, L.; HAMMAR, N.; HOGSTEDT, C. Incidence of myocardial infarction and mortality from specific causes among bus drivers in Sweden. Int. J. Epidemiol. England, v. 22, n. 1, p. 57-61, Dec. 1993.

ANJOS, L. A. Índice de massa corporal (massa corporal/estatura ${ }^{2}$ ) como indicador do estado nutricional de adultos: revisão da literatura. Rev. Saúde públ., São Paulo, v. 26, n. 6, p. 431-436, dez. 1992.

BACKMAN, A. L.; JARVINEN, E. Turnover of professional drivers. Scand. J. Work Environ. Health, Finland, v. 9, n. 1, p. 36-41, Feb. 1983.

BEIGEL, A. et al. Planning for the development of comprehensive community alcoholism services: I. the prevalce survey. Am. J. Psychiatry, United States, v. 131, n. 10, p. 1112-1116, Oct. 1974.

BENAVIDES, F. G. et al. Occupational categories and sickness absence certified as attributable to common diseases. Eur. J. Public Health, England, v. 13, n. 1, p. 51-55, Mar. 2003.

BIGERT, C. et al. Myocardial infarction among professional drivers. Epidemiology, v. 14, n. 3, p. 333339, May 2003.

CHECKOWAY, H.; PEARCE, N. E.; KRIEBEL, D. Research methods in occupational epidemiology. 2. ed. New York: Oxford University Press, 2004. 392 p.

CORDEIRO, R.; LIMA-FILHO, E. C.; NASCIMENTO, L. C. Associação da perda auditiva induzida pelo ruído com o tempo acumulado de trabalho entre motoristas e cobradores. Cad. Saúde públ., Rio de Janeiro, v. 10, n. 2, p. 210-221, jun. 1994.

CORDEIRO, R. et al. Associação da pressão arterial diastólica com o tempo acumulado de trabalho entre motoristas e cobradores. Rev. Saúde públ., São Paulo, v. 27, n. 5, p. 363-372, out. 1993.

CORREA FILHO, H. R. et al. Perda auditiva induzida por ruído e hipertensão em condutores de ônibus. Rev. Saúde públ., São Paulo, v. 36, n. 6, p. 693-701, dez. 2002.
COSTA, L. B. et al. Morbidade declarada e condições de trabalho: o caso dos motoristas de São Paulo e Belo Horizonte. São Paulo em Perspec., São Paulo, v. 17, n. 2, p. 54-67, jun. 2003.

COTTINGTON, E. et al. Psychosocial factors and blood pressure in the Michigan statewide blood pressure survey. Am. J. Epidemiol., United States, v. 121, n. 4, p. 515-519, Apr. 1985.

DÓREA, E. L.; LOTUFO, P. A. Epidemiologia da hipertensão arterial sistêmica. Rev. Hipertens., São Paulo, v. 7, n. 3, p. 86-89, 2004.

GARRIDO, M. J.; FERNANDEZ-GUINEA, S. Neuropsychological deficits in alcoholics: some implications for road safety. Rev. Neurol., Spain, v. 38, n. 3, p. 277-283, June 2004.

GIMENO, D. et al. External validation of psychological job demands in a bus driver sample. J. Occup. Health., Japan, v. 46, p. 43-48, Jan. 2004.

GUS, I. et al. Prevalência, reconhecimento e controle da hipertensão arterial sistêmica no estado do Rio Grande do Sul. Arq. bras. Cardiologia, Rio de Janeiro, v. 83, n. 5, p. 424-428, nov. 2004.

HARTVIG, P.; MIDTTUN, O. Coronary heart disease risk factors in bus and truck drivers. A controlled cohort study. Int. Arch. Occup. Environ. Health, Germany, v. 52, n. 4, p. 353-360, 1983.

INSTITUTO BRASILEIRO DE GEOGRAFIA E ESTATISTICA. Censo demográfico de 2000. Rio de Janeiro, 2001.

ISSEVER, H. et al. Personality characteristics, psychological symptoms and anxiety levels of drivers in charge of urban transportation in Istanbul. Occup. Med., England, v. 52, n. 6, p. 297-303, Sept. 2002.

KLEINBAUM, D. et al. Applied regression analysis and other multivariable methods. 3. ed. Pacific Grove: Duxbury Press, 1998.

KLOETZEL, K. et al. Relationship between hypertension and prolongued exposure to heat. $J$. Occup. Med., United States, v. 15, n. 11, p. 878-885, Nov. 1973.

LÓLIO, C. A. de et al. Hipertensão arterial e possíveis fatores de risco. Rev. Saúde públ., São Paulo, v. 27, n. 5, p. 357-362, out.1993.

MARI, J. J.; WILLIAMS, P. A. Validity study of a psychiatric screening questionnaire (SRQ-20) in 
primary care in the city of São Paulo. Br. J. Psychiatry, England, v. 148, p. 23-26, Jan. 1986.

MARTINS, P. J. et al. Increased plasma homocysteine levels in shift working bus drivers. Occup. Environ. Med., England, v. 60, n. 9, p. 662-666, Sept. 2003.

MELLO, M. T. et al. Sleep patterns and sleep-related complaints of Brazilian interstate bus drivers. Braz. J. Med. Biol. Res., Brazil, v. 33, n. 1, p. 71-77, Jan. 2000.

MICHAELS, D.; ZOLOTH, S. R. Mortality among urban bus drivers. Int. J. Epidemiol., England, v. 20, n. 2, p. 399-404, June 1991.

MORRIS, J. N. et al. Incidence and prediction of ischemic heart disease in London busmen. Lancet, England, v. 2, n. 7463, p. 553-559, Sept. 1966.

NETTERSTROM, B.; SUADICANI, P. Self-assessed job satisfaction and ischaemic heart disease mortality: a 10-year follow-up of urban bus drivers. Int. J. Epidemiol., England, v. 22, n. 1, p. 51-56, Feb. 1993.

OLIVEIRA, A. C. F. de; PINHEIRO, J. Q. Indicadores psicossociais relacionados a acidentes de trânsito envolvendo motoristas de ônibus. Psicologia Est., Maringá, v. 12, n. 1, p. 171-178, abr. 2007.

PAES-MACHADO, E.; LEVENSTEIN, C. Assaltantes a bordo: violência, insegurança e saúde no trabalho em transporte coletivo de Salvador, Bahia, Brasil. Cad. Saúde públ., Rio de Janeiro, v. 18, n. 5, p. 1215-1227, set./out. 2002.

PICCINI, R. Hipertensão arterial sistêmica em Pelotas, $R S$ : prevalência, fatores de risco e manejo. 1993. 152 f. Dissertação (Mestrado em Epidemiologia) - Universidade Federal de Pelotas, Pelotas, 1993.

RAGLAND, D. R. et al. Hypertension and years of driving in transit vehicle operators. Scand. J. Soc. Med., Sweden, v. 25, n. 4, p. 271-279, Dec. 1997.
ROSENGREN, A.; ANDERSON, K.; WILHELMSEN, L. Risk of coronary heart disease in middle-aged male bus and tram drivers compared to men in other occupations: a prospective study. Int. J. Epidemiol., England, v. 20, n. 1, p. 82-87, Mar. 1991.

RYDSTEDT, L. W.; JOHANSSON, G.; EVANS, G. W. The human side of the road: improving the working conditions of urban bus drivers. J. Occup. Health Psychol., United States, v. 3, n. 2, p. 161-171, Apr. 1998.

SANTOS, E. H. et al. Sleep and sleepiness among brazilian shift-working bus drivers. Chronobiol. Int., United States, v. 21, n. 6, p. 881-888, 2004.

SOUZA, M. de F. M.; SILVA, G. R. da. Risco de distúrbios psiquiátricos menores em área metropolitana na região sudeste do Brasil. Rev. Saúde públ., v. 32, p. 50-58, fev. 1998.

VANDERVOORT, D. J.; RAGLAND, D. R.; SYME, S. L. Anger expression and hypertension in transit workers. Ethn. Dis., United States, v. 11, n. 1, p. 8089, 2001.

WAHLBERG, A. E. The stability of driver acceleration behavior, and a replication of its relation to bus accidents. Accid. Anal. Prev., England, v. 36, n. 1, p. 83-92, Jan. 2004.

WANG, P. D.; LIN, R. S. Coronary heart disease risk factors in urban bus drivers. Public Health, England, v. 115, n. 4, p. 261-264, July 2001.

WINKLEBY, M. A. et al. Excess risk of sickness and disease in bus drivers: a review and synthesis of epidemiological studies. Int. J. Epidemiol., England, v. 17, n. 2, p. 255-262, June 1988a.

WINKLEBY, M. A. et al. Heightened risk of hypertension among black males: the masking effects of covariables. Am. J. Epidemiol., United States, v. 128, n. 5, p. 1075-1083, Nov. 1988b. 